OPEN ACCESS

Edited by:

Shengjun Wang,

Jiangsu University, China

Reviewed by:

Philipp E. Scherer,

University of Texas Southwestern

Medical Center, United States

Wendy B. Bollag,

Augusta University, United States

${ }^{*}$ Correspondence:

Xin Su

13875905699@163.com

Dong Chang

changdongiheart@163.com

Specialty section: This article was submitted to Autoimmune and Autoinflammatory Disorders, a section of the journal

Frontiers in Immunology

Received: 11 August 2020 Accepted: 14 December 2020 Published: 01 February 2021

Citation:

Su X, Cheng Y and Chang D (2021)

The Important Role of Leptin in

Modulating the Risk of

Dermatological Diseases.

Front. Immunol. 11:593564. doi: 10.3389/fimmu.2020.593564

\section{The Important Role of Leptin in Modulating the Risk of Dermatological Diseases}

\author{
Xin Su*, Ye Cheng and Dong Chang * \\ Department of Cardiology, The Xiamen Cardiovascular Hospital of Xiamen University, Xiamen, China
}

It is an indisputable fact that obesity is associated with a series of health problems. One important hallmark of obesity is excessive accumulation of lipids in the adipocyte, especially triglyceride (TG). Currently, the adipocyte has been considered not only as a huge repository of excess energy in the form of fat but also as an important source of multiple hormones and cytokines called adipokines. In obesity, the adipocyte is dysfunctional with excessive production and secretion of pro-inflammatory adipokines, such as tumor necrosis factor $\alpha(\mathrm{TNF}-\alpha)$, interleukin 6 (IL-6), and leptin. On the other hand, accumulating evidence has shown that leptin plays a vital role in stimulating angiogenesis, controlling lipid metabolism, and modulating the production of pro-inflammatory cytokines. Furthermore, the various activities of leptin are related to the wide distribution of leptin receptors. Notably, it has been reported that enhanced leptin levels and dysfunction of the leptin signaling pathway can influence diverse skin diseases. Recently, several studies revealed the roles of leptin in wound healing, the hair cycle, and the pathogenic development of skin diseases, such as psoriasis, lupus erythematosus, and dermatological cancers. However, the exact mechanisms of leptin in modulating the dermatological diseases are still under investigation. Therefore, in the present review, we summarized the regulatory roles of leptin in the pathological progression of diverse diseases of skin and skin appendages. Furthermore, we also provided evidence to elucidate the complicated relationship between leptin and different dermatological diseases, such as systemic lupus erythematosus (SLE), psoriasis, hidradenitis suppurativa, and some skin tumors.

Keywords: leptin, obesity, immune system, leptin receptor, dermatological diseases

\section{INTRODUCTION}

Obesity, defined as having a body mass index (BMI) greater than $30 \mathrm{~kg} / \mathrm{m}^{2}$, is associated with numerous health problems that include the combination of insulin resistance, hypertension, and cardiovascular disease (CVD) (1). Worldwide, the prevalence of obesity has approximately doubled during the past several decades, resulting in multiple obesity-related pathological conditions and posing serious risks to future health (2).

Notably, one important hallmark of obesity is excessive accumulation of lipids in the adipocyte, especially triglyceride (TG). Currently, the adipocyte has been considered not only as a huge 
repository of excess energy in the form of fat but also as an important source of multiple hormones and cytokines, named adipokines (3). In obesity, the adipocyte is dysfunctional with excessive production and secretion of pro-inflammatory adipokines, such as tumor necrosis factor $\alpha$ (TNF- $\alpha$ ), interleukin 6 (IL-6), and leptin (4). Accumulating evidence has shown the role of these adipokines in multiple pathophysiological processes, including energy homeostasis, lipid metabolism, immunological activity, and the development of dermatological diseases.

Leptin, a $16-\mathrm{kDa}$ protein encoded by the obese gene on chromosome 7q31.3, is mainly synthesized and secreted by subcutaneous adipose tissue (5). As shown in previous studies, circulating levels of leptin are strongly associated with total body weight in mice, suggesting that alterations of leptin concentration play an important role in modulating the process of energy intake and lipid metabolism (6). On the other hand, due to a wide distribution of the leptin receptor, it is proposed that leptin possesses diverse functions which affect multiple biological processes in different tissues. Consistent with this notion, it has been shown that leptin plays a vital role in stimulating angiogenesis, controlling lipid metabolism, and modulating the production of pro-inflammatory cytokines (7).

It is worth noting that patients with skin diseases have a higher risk of subclinical metabolic syndrome and CVD compared to that in individuals without skin disease. Indeed, it is accepted that diverse skin diseases, such as psoriasis, lichen planus, connective tissue diseases, bullous diseases, vitiligo, and chronic urticarial diseases, are closely associated with cardiometabolic disorders (8). However, the underlying mechanisms are still not elucidated. Concerning this notion, focus is recently shifting towards elucidating potential mechanisms whereby different adipokines regulate the pathological progression of skin diseases (9). Consistently, a vital role of leptin has begun to gain appreciation, but the exact function is still unclarified. According to recent studies, leptin influences dermatological pathophysiology and as a consequence, might have an important impact on skin diseases and other systemic autoimmune disorders. Therefore, in the present review, we summarized the vital role of leptin in the pathological progression of diverse diseases of skin and skin appendages. Furthermore, we also provided evidence to elucidate the complicated relationship between leptin and different skin diseases, such as systemic lupus erythematosus (SLE), psoriasis, hidradenitis suppurativa, and some skin tumors.

\section{RELATIONSHIP BETWEEN OBESITY AND DIVERSE SKIN DISEASES}

Taking into account the growing number of obese individuals and unquestionable participation of adiposity in many pathological processes, we could reasonably speculate that increased body fat mass may result in the disruption of normal processes in the skin. As reported, obesity is considered to be a status of chronic and low-grade inflammation which leads to the disturbed secretion of multiple cytokines, such as leptin, adiponectin, and chemokines, that play a modulatory role in inflammatory response (10). Actually, in the obese condition, the adipocyte is dysfunctional with aberrant production of proinflammatory adipokines, including tumor necrosis factor $\alpha$ (TNF- $\alpha$ ), interleukin (IL)-6, leptin, visfatin, resistin, angiotensin II, and plasminogen activator inhibitor 1 (PAI-1). Noteworthy, the excessive pro-inflammatory adipokines within the circulation can presumably stimulate inflammatory signaling pathways and affect the development of obesity in turn. Thereby, leptin is currently regarded as the most important proinflammatory adipokine, since it stimulates the production of IL-1, IL-6, IL-12, and TNF- $\alpha$ by innate immune cells and enhances reactive oxygen species (ROS) production $(11,12)$.

On the other hand, systemic adiposity also results in alterations of dermatological physiology. Therefore, it has been proposed that obesity could be considered as one of the most vital risk factors for the development of several dermatological diseases (13). Indeed, obesity is confirmed to be associated with venous stasis, lymphedema, and an increased rate of infection, such as candidiasis, intertrigo, candida folliculitis, furunculosis, erysipelas, cellulitis, erythrasma, tinea cruris, folliculitis, and necrotizing fasciitis. Otherwise, the obese condition also increases the risk of selected inflammatory dermatoses, such as psoriasis, hidradenitis suppurativa, and atopic dermatitis (14).

Some dermatological abnormalities, such as acanthosis nigricans, keratosis pilaris, striae diseases, skin tags, and palmoplantar keratodermas, have been observed more commonly in obese patients than in those individuals with normal BMI $(14,15)$. With the development of obesity, elevated concentrations of hormones, including androgens, insulin, growth hormone, and insulin-like growth factor, could induce the escalation of sebum production which subsequently exacerbate acne (8). Therefore, according to published results, immune dysregulation and elevated levels of pro-inflammatory adipokines, in particular leptin, have a significant function in regulating skin biology and the risk of multiple dermatological diseases $(16,17)$.

\section{POTENTIAL MECHANISMS OF LEPTIN IN SYSTEMIC METABOLISM}

Leptin is synthesized and secreted mainly by the white adipose tissue (WAT). However, a small amount of leptin is produced by the hypothalamus, pituitary, gastric mucosa, bone marrow, mammary epithelium, skeletal muscle, and placenta (7). Due to the technological advances, major breakthroughs have been made to explain biological function of leptin.

Leptin acts as a kind of pleiotropic hormone. Under obese or overweight conditions, the secretion of leptin is increased significantly. In addition, multiple other factors, such as insulin, glucose, estrogens, and diverse adipokines might also enhance the secretion of leptin (18). Interestingly, the peripheral leptin level is shown to follow a circadian rhythm with a peak at night. Furthermore, given that the serum leptin concentration is strongly 
correlated with the amount of body fat mass and the BMI value, some scholars have proposed that obesity should be characterized by enhanced circulating leptin levels (5). More recently, an independent research demonstrated that higher serum leptin concentrations coexisted with leptin resistance, and the disturbance was closely related to the development of obesity (19).

Aside from the established role of leptin in modulating the development of several dermatological diseases, the function of leptin receptors has also been given substantial attention in recent years. The expression of leptin receptor was found in the hypothalamus, fibroblasts, endothelial cells, keratinocytes, adipocytes, and blood mononuclear cells (20). After binding to its receptor in the hypothalamus, leptin activates an important signaling cascade which subsequently induces the inhibition of several orexigenic neuropeptides (21). Thus, we can speculate from these findings that the wide distribution of leptin receptors leads to the pleiotropic function of leptin.

Leptin receptor is a transmembrane receptor which is similar to the Class I cytokine receptors family (22). Owing to the differences within the basic structure, several isoforms of leptin receptor can be distinguished, including the short isoform and the full-length isoforms. To expand something in detail, the short isoforms of the leptin receptor comprise leptin receptor-A, leptin receptor-C, leptin receptor-D, and leptin receptor-F. The fulllength isoform of leptin receptor contains leptin receptor-B (23). Notably, the full-length isoform of the leptin receptor is also considered to be responsible for controlling food intake and systemic energy homeostasis. Two short isoforms of leptin receptor, leptin receptor-A and leptin receptor-C, are located predominantly in micro-vessels within the central nervous system (CNS) where they are responsible for leptin circulation in the cerebrospinal fluid, as well as receptor-mediated transport of leptin through the blood-brain barrier (24). Additionally, leptin receptor-A and leptin receptor-C in extra-neural tissues have been verified to determine the functional pleiotropy of leptin; whereas the soluble isoform, leptin receptor-E, provides binding variety and bioavailability of leptin (25).

The important role of leptin in regulating intracellular signaling pathways has also begun to gain appreciation. According to published reports, leptin can stimulate multiple signaling pathways including the Janus kinase/signal transducer and activator of transcription (JAK/STAT) pathway, the phosphoinositide 3-kinase (PI3K) pathway, the mitogenactivated protein kinase (MAPK) pathway, the extracellular signaling-regulated kinase $1 / 2(\mathrm{ERK} 1 / 2)$ pathway, the adenosine monophosphate kinase (AMPK) pathway, and the PPAR gamma coactivator/peroxisome proliferator-activated receptor (PGC/PPAR) pathway (26). Among these important signaling pathways, the JAK/STAT signal transduction cascade is the most important signaling pathway that can be activated by leptin. After binding of leptin with the full-length isoform of the leptin receptor, JAK is phosphorylated activated which subsequently promotes the phosphorylated stimulation of STAT3, leading to its dimerization followed by migration to the nucleus where STAT3 influences the expression of target genes, such as the suppressor of cytokine signaling 3 (SOCS3) (27). The comprehensive signaling and function of leptin and the leptin receptor are shown in Figure 1.

Recently, leptin has been found to play a role in modulating mitochondrial metabolism since it increases the efficiency of

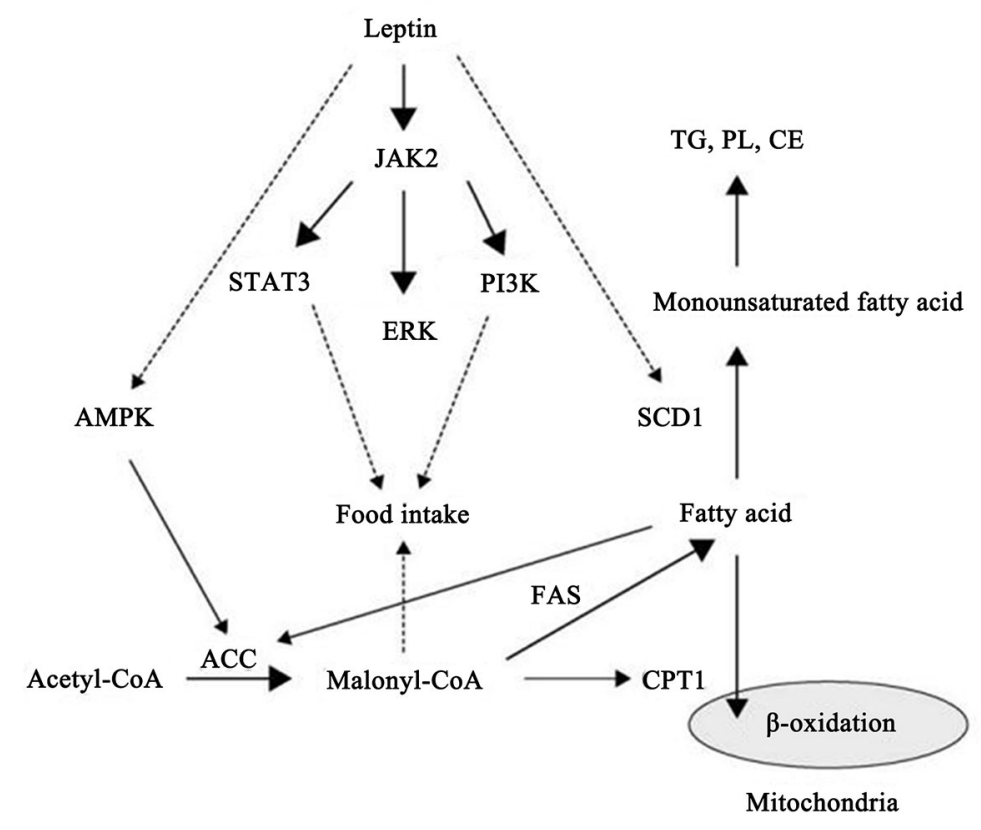

FIGURE 1 | Schematic representation of leptin and leptin receptor signal transduction pathways and functions. Stimulation of the leptin receptor by leptin can activate JAK2 kinase, resulting in tyrosine phosphorylation of the receptor and downstream proteins, including STAT3, SHP2, IRS2, and PI3K, that play an important role in regulating transcription of genes essential for energy intake and lipid metabolism. 
oxidation-reduction reactions and energy utilization within mitochondria (28). Taken together, the results summarized above could provide the potential mechanisms by which leptin and its receptor regulates the development of diverse skin diseases.

\section{BIOLOGICAL FUNCTIONS OF LEPTIN}

Given that the biological functions of leptin are being extensively elucidated, the focus of this section is to discuss recent research elucidating the main functions of leptin. Under physiological condition, leptin exerts the ability to limit food intake, control body fat mass, and stimulate energy expenditure by negative feedback at the hypothalamic nuclei (29). However, in obesity status, the dysfunctional adipocyte may lead to an irrepressible increase in circulating leptin (30). This phenomenon might be a consequence of the dysfunction of leptin signaling pathways which may inhibit the combination of leptin with its receptors, followed by changes in leptin receptor expression or signal transduction (31).

On the other hand, it has been demonstrated that leptin also affects the immune system by acting as a pro-inflammatory adipokine. Indeed, as mentioned above, leptin itself activates the secretion of several other pro-inflammatory cytokines, as well as increases nitric oxide (NO) release and stimulates phagocytosis by monocytes/macrophages (32). Likewise, in neutrophils, leptin is also confirmed to induce the synthesis of oxygen free radicals, playing an essential role in the oxidative stress response. Recently, emerging evidence showed that leptin enhanced the cytotoxicity and proliferation of natural killer (NK) cells. Aside from the effects mentioned above, leptin also has the property to activate the chemotaxis of eosinophils, basophils, and neutrophils (33). It is noteworthy that leptin increases the production of several pro-inflammatory cytokines, such as IL8, IL-12, IL-6, and TNF- $\alpha$, from dendritic cells. Furthermore, by influencing lymphocyte receptors, leptin modulates the balance of Th1/Th2 lymphocytes towards the Th1 lymphocyte phentype, which subsequently leads to an aggravation of the inflammatory response. The wide impact of leptin on the immune system indicates the important role of leptin in the pathogenic development of immune diseases (34). The multiple effects of leptin on different types of immune cells of the innate and adaptive immune systems is shown in Figure 2.

\section{THE IMPACT OF LEPTIN ON HAIR AND DIVERSE SKIN DISEASES}

Although the adipocyte isolated from subcutaneous tissue is a prevalent site of leptin synthesis, it has been reported that fibroblasts and keratinocytes also possess the ability to produce leptin and express leptin receptors (35). With in-depth investigation, the expression of the leptin receptor has been detected in the epidermis, predominantly in the basal layer and the hair follicle papilla cells (36). Interestingly, results showed that leptin possessed the ability to stimulate the proliferation of keratinocytes and fibroblasts, as well as to facilitate epithelialization and collagen synthesis. These processes lead to an improvement in skin regeneration (37). Moreover, the local synthesis and secretion of leptin increases significantly after skin injury which results in shortening of the period of wound healing, suggesting an important role of leptin in skin regeneration (38). Additionally, independent research revealed that leptin also supported the skin micro-organism defense,

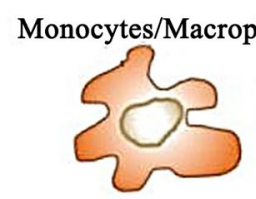

TLR $2 \uparrow$

IL-6, TNF- $\alpha$, IL- $1 \beta \uparrow$

Phagocytosis $\uparrow$
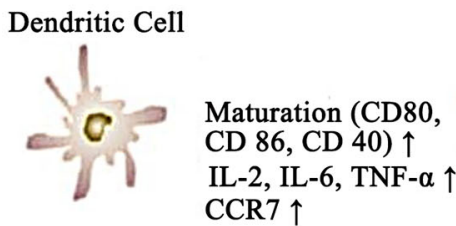

NK Cell

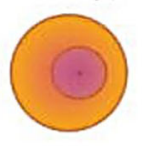

TRAIL $\downarrow$

CD107a $\downarrow$
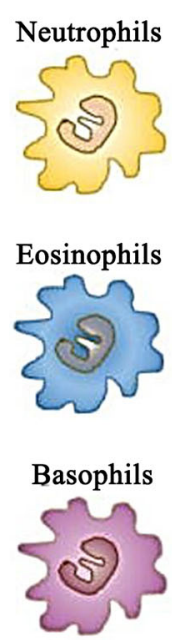

Chemotaxis $\uparrow$

IL-4, IL-13 $\uparrow$

Innate Immunity

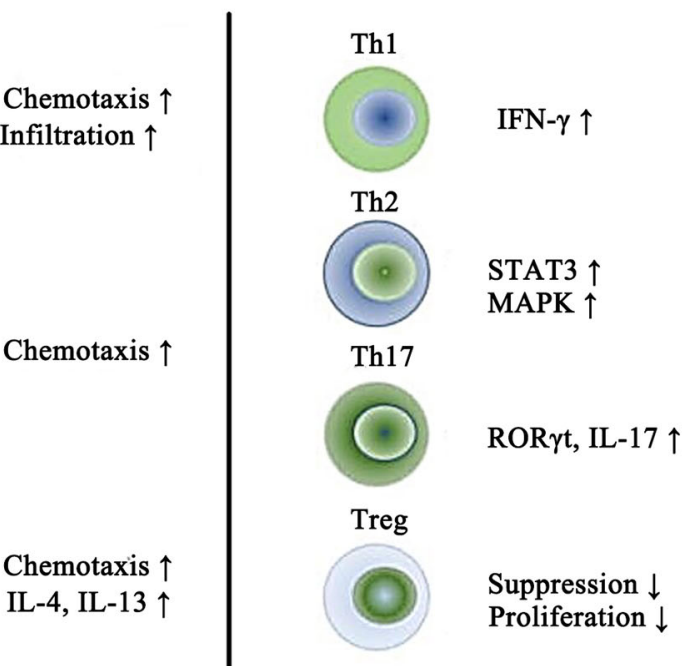

Adaptive Immunity

FIGURE 2 | Schematic representation of the effects of leptin on different immune cells of the innate and adaptive immune systems. 
which provided another potential mechanism whereby leptin regulated the wound healing process (39). However, by stimulating the STAT3 signaling pathway, leptin could trigger the proliferation, differentiation, and migration of diverse cell types in the skin, as well as modulating the pathological process of angiogenesis (40).

In 2018, Lee et al. conducted a research and demonstrated the molecular mechanism by which leptin modulated the proliferation of keratinocytes via examining the genome-wide transcriptional responses of normal human keratinocytes (NHKs) (35). In addition, leptin was found to enhance several intracellular signaling pathways, such as the PI3K signaling pathway, and induced a pro-inflammatory response in the keratinocyte. Enhanced function of leptin was induced by the increased production of interleukins in a similar mechanism as was observed within immune cells. Taken together, the above effects of leptin might have a significant impact on the risk and development of dermatological diseases and related chronic metabolic disorders $(41,42)$.

\section{Relationship Between Leptin and Hair}

Owing to technological advances, several eye-catching breakthroughs have been put forward to further illuminate the association between leptin and hair. According to novel published results, immuno-histochemical analyses revealed the presence of leptin protein and leptin mRNA in different hair structures, including the matrix, the inner root sheath, and the follicular dermal papilla (43). Noticeably, in mice with leptin deficiency (the ob/ob mouse model), the period of the first anagen cycle was found to be delayed significantly. The authors suggested that leptin could be considered as an essential activator of anagen to promote the hair growth (43). Similarly, the injection of exogenous leptin stimulated anagen conversion in resting hair follicles (44). Nevertheless, the exact mechanism of the influence of leptin on the hair cycle and hair growth is still not fully elucidated (45). We still need further investigation to explore the relationship between leptin and the process of hair growth.

\section{Relationship Between Leptin and Psoriasis}

Psoriasis is one of the most common skin diseases with a multifactorial pathogenesis. The inflammatory process underlying the skin changes usually has three features, including erythema, thickening, and scale. The typical psoriatic skin lesions present as silver-whitish scales with sharply demarcated, red, and thickened areas (46). According to the results of epidemiological investigation, the prevalence of psoriasis is various in different regions, but overall it reaches approximately $2 \%$ of the world population (47).

Recently, psoriasis has been considered to be a kind of genetic-, autoimmune-, and metabolic-derived disease. It has been speculated that when a genetically predisposed individual is exposed to specific environmental factors (EFs) that act along with epigenetic alternations, he/she may present psoriatic skin alterations $(48,49)$. Well-known EFs that are strongly linked to psoriasis include dietary habits along with obesity, microbiota, infections, alcohol intake, tobacco smoking, and psychological factors $(50,51)$.

There are numerous studies concerning serum adipokine levels during the course of psoriasis, and these observations comprise not only leptin but also resistin, adiponectin, and other inflammatory cytokines $(52,53)$. In addition, higher levels of leptin within the sera of psoriatic patients were observed in comparison with those in control participants (54, 55). On the other hand, remarkably increased leptin levels were found in obese individuals, particularly in those with obesity, suggesting a positive relationship between elevated levels of leptin and the risk of psoriasis and metabolic disorders (56). Interestingly, this finding was further replicated in another investigation conducted by Mitsuyama and colleagues. According to this report, the authors demonstrated that the expression of leptin mRNA was significantly enhanced in obese and psoriatic individuals compared to that in non-obese counterparts (57). Of note, some studies demonstrated robustly decreased levels of leptin in peripheral tissues after systemic therapy for psoriasis, such as using cyclosporine-A, suggesting a close relationship between serum levels of leptin and the pathological progression or regression of psoriasis (58).

Recently, a positive correlation between serum leptin concentration and the severity of psoriasis, which is evaluated by the Psoriasis Area and Severity Index (PASI), was also put forward (59). Using the ob/ob mice with imiquimod (IMQ)induced psoriasis, Stjernholm et al. demonstrated that these mice exhibited attenuated manifestations of inflammation, including erythema, infiltration, and scaling in dorsal and ear skin (60). On the other hand, Shimoura et al. observed that IMQ-induced psoriatic-related inflammation was exacerbated in leptin receptor-deficient diabetic obese mice ( $\mathrm{db} / \mathrm{db}$ mice) compared with that in control mice, accompanied by significantly elevated expression of C-X-C motif ligand 5 (CXCL5) and IL-6. Importantly, massive number of CXCL5 positive cells infiltrated into the dermis and subcutaneous adipose tissue of the diabetic obese mice, suggesting a function of inflammatory cytokines in the increase of psoriasis severity with concomitant cardio-metabolic disorders (61). Due to its pro-inflammatory activity, leptin promoted production of IL-1, IL- 6 , and TNF- $\alpha$, which also impacted the development and the severity of psoriasis (62). Notably, all the above-mentioned processes could stimulate the Th1/Th17 lymphocyte axis and induce a higher concentration of IL-17/IL-23 (63). Moreover, leptin is also confirmed to be involved in the activation of the Th1/Th17 lymphocyte axis via the JAK/STAT3 signaling pathway, which could further exaggerate angiogenesis in psoriatic lesions (64).

Recently, it has been suggested that enhanced levels of leptin in patient's skin may induce the production of amphiregulin, which is an epidermal growth factor receptor ligand with a probable role in promoting keratinocyte proliferation (65). Furthermore, concentrations of CXCL8, which can also induce keratinocyte proliferation, were found to be increased remarkably in the skin of psoriatic patients (66). It is worth noting that leptin may also stimulate the production of CXCL8 by monocytes (67). According to these findings, we can 
reasonably speculate that leptin links obesity and psoriasis potentially via modulating the expression level of diverse chemokines within the circulation.

On the other hand, since alterations caused by mutation of the LEPTIN gene have been less addressed, the focus of the current paragraph is to discuss the recent work describing the relationship between single nucleotide polymorphisms (SNPs) of the LEPTIN gene and the development of psoriasis. For instance, the group of Torres et al. used the data from a Genome Wide Association Study (GWAS) and found that two important SNPs, including the rs2167270 SNP and the rs1137100 SNP, had no significant relationship with the development of arteriosclerosis and obesity in a cohort of psoriatic patients (68). Similar to these findings, results from research conducted by Karpouzis et al., who examined the rs2060713 SNP, did not establish any link between the SNP and the severity of psoriasis (69). In contrast, Abdel Hay et al. observed that participants who carried the G2548A SNP presented elevated circulating levels of leptin as well as an increased risk of psoriasis, indicating that the G2548A SNP may be a novel predictor for the development of psoriasis (70).

However, more large-scale clinical investigations are still needed to further elucidate the comprehensive relationship between diverse SNPs of LEPTIN gene and the risk and severity of psoriasis.

\section{Relationship Between Leptin and Systemic Sclerosis}

The lack of activation of the STAT3 signaling pathway has been shown to lead to multiple important alterations in the skin (71). Therefore, the question could be raised as to whether there is a link between insufficient activation of leptin signaling pathways and dermatological fibrotic diseases.

Several meta-analyses showed that in patients suffering from systemic sclerosis, serum leptin levels were comparable to those of healthy control individuals. For instance, to determine the relationship between serum leptin levels and disease activity, Budulgan et al. conducted a study enrolling 30 healthy individuals and 30 patients with systemic sclerosis. After analysis, the authors demonstrated that there was no significant difference between the two groups in terms of serum leptin levels. Nevertheless, the serum leptin levels were significantly reduced in patients with active systemic sclerosis, suggesting that leptin could be used as an activity biomarker in patients with this disease (72). Likewise, these findings were replicated in another study conducted by Olewicz-Gawlik and colleagues. According to these results, the authors also found no statistically significant difference in circulating levels of leptin between patients with systemic sclerosis and healthy control individuals. However, circulating concentrations of leptin were shown to be correlated with the duration of symptoms (73). A meta-analysis containing 14 studies also showed similar results (74). Thus, it seems that serum levels of leptin are not significantly different between patients with systemic sclerosis and healthy individuals.
On the contrary, several studies showed that the serum levels of leptin increased in patients with systemic sclerosis. In 2012, Pehlivan et al. showed that circulating leptin levels were significantly increased by approximately 5 -fold in patients with systemic sclerosis compared with those in healthy controls (75). More recently, another study which enrolled 100 patients with confirmed systemic sclerosis diagnosis and 20 healthy individuals revealed that the patients presented significantly elevated serum levels of leptin (76). The discordant results summarized above might be attributed to diverse age, sex, and race of the enrolled participants. Further studies, including largescale study of many subjects, should be carried out to clarify this relationship.

The underlying mechanism whereby leptin facilitates the development of systemic sclerosis could be also attributed to the function of leptin in indirectly stimulating the development of fibrosis. It has been well demonstrated that binding of leptin to its receptor in regulatory $\mathrm{T}$ lymphocytes is a signal for decreased proliferation of regulatory $\mathrm{T}$ lymphocytes which could enhance fibrosis (77). In addition, leptin has also been suggested to promote fibrosis by an alternative mechanism related to the renin-angiotensin system (RAS). What is more, in the elderly population, leptin is shown to be associated with arterial stiffness (78), which is one of the features of vascular fibrosis. Accordingly, leptin might be useful as a potential marker of vascular damage in systemic sclerosis.

\section{Relationship Between Leptin and Systemic Lupus Erythematosus}

Systemic lupus erythematosus (SLE) is characterized by excessive accumulation of anti-nuclear auto-antibodies, hyper-activation of immune cells, and aberrant infiltration of $\mathrm{T}$ lymphocytes, leading to the systemic damages triggered by immune complexes deposition (79). Importantly, with more advanced disease, several other organs and systems, including the renal, pulmonary, cardiovascular, and central nervous systems, are also affected. In terms of the responses of immune cells, the defective erasure of auto-reactive lymphocytes, the immune responses against autoantigens, and the loss of self-tolerance, are also vital contributing factors to susceptibility of SLE.

It has already been confirmed that serum levels of leptin increased significantly in patients with SLE, a finding that has been replicated in many studies from diverse countries, revealing that leptin can be considered as a novel biomarker in predicting the risk of SLE (80-83). Recently, several possible mechanisms by which leptin modulates the development of SLE have been emphasized. For instance, using mouse models, it was shown that leptin promoted the differentiation of Th17 lymphocytes and enhanced synthesis of IL-17 via interacting with the RARrelated orphan receptor gamma (ROR- $\gamma$ ) (84). Moreover, leptin could function together with another pro-inflammatory factor, neutrophil-activating protein (NAP-2), and activate the PI3K/ Akt signaling pathway in SLE patients (85). On the other hand, leptin promoted survival and proliferation of auto-reactive $\mathrm{T}$ lymphocytes in mice with an SLE-like mutation (86). An 
inhibiting effect of leptin on T regulatory lymphocytes was also suggested (77).

Leptin could influence immune cell subsets, modulate cytokine secretion, and promote anti-apoptotic protein expression. Nevertheless, the interaction between leptin and other inflammatory cells under SLE condition is not fully understood (87). Additionally, no significant difference was observed between patients with active and those with inactive SLE (80, 88, 89). Nevertheless, a lower leptin concentration was found with joint inflammation and neurological symptoms during a course of SLE (90). More studies, including large-scale clinical trials and animal research, are still needed to clarify the relationship between leptin and SLE and the underlying mechanisms.

\section{Relationship Between Leptin and Skin Tumors}

As described in previous studies, in obesity, dysfunctional adipocytes expressed excessive leptin which subsequently increased the risk of many skin tumors (91-93). Recently, it was also demonstrated that an increased leptin concentration may accelerate the growth of melanoma, to increase mortality in patients $(94,95)$. Recent research has also shown a positive correlation between serum leptin levels and the number of skin tags. Furthermore, either the higher circulating levels of leptin or the reduced levels of leptin receptors can trigger the proliferation and differentiation of keratinocytes into skin tag lesions $(96,97)$. Taken together, these findings highlight the function of leptin in promoting the development of dermatological tumors.

\section{Relationship Between Leptin and Hidradenitis Suppurativa}

According to the latest results, an important relationship between leptin and hidradenitis suppurativa has begun to gain appreciation since leptin plays an important role in proinflammatory responses. To expand, it has been found that obesity is an independent risk factor for hidradenitis suppurativa, and the risk for patients with hidradenitis suppurativa to suffer from obesity and metabolic syndrome is relatively higher compared to that in healthy controls. Consistent with this notion, Malara and colleagues observed that serum levels of leptin were significantly increased in patients with hidradenitis suppurativa (98). On the other hand, it should be emphasized that enhanced local concentrations of leptin in subcutaneous adipose tissue could cause an intensification of the inflammatory response within the skin of patients with hidradenitis suppurativa. This marked alteration may subsequently result in an increase of systemic inflammation and exacerbate the symptoms of hidradenitis suppurativa (99). The potential role of leptin in selected skin diseases is summarized and presented in Table 1.

\section{CONCLUSIONS AND PERSPECTIVES}

Leptin is a pluripotent adipokine and is strongly correlated with multiple immune responses in humans. The pleiotropic effects of leptin in modulating the pathogenic development of various skin diseases have been well demonstrated. As a consequence of adiposity and increased leptin levels, especially in conjunction with reduced expression of leptin receptors, several pathological processes have been observed in the skin and the skin appendages. Although the potential mechanisms whereby leptin modulates disease development have been thoroughly investigated, the exact role of leptin in dermatological disorders still needs to be further elucidated.

On the other hand, it is a challenge to explore all of the potential confounding factors which affect dermatological pathology. Moreover, it is also important to further find out the exact mechanism whereby those factors affect skin and skin appendages. It needs to be highlighted that not all activities of leptin in the skin are fully understood. Another unsolved problem is that there is no clear explanation of why some obese individuals present with a worse course of skin diseases. It is worth noting that the recently postulated concept for leptin, "the less leptin is more" in the obese setting, may offer a better explanation for this issue. Otherwise, it is still an open question of whether pharmacological intervention resulting in decreased leptin secretion or breaking the leptin resistance could be a target for the treatment of different skin diseases. Actually, several leptin antagonists and leptin-neutralizing antibodies have been developed and could provide possible therapies for treating leptin-related dermatological diseases.

TABLE 1 | The potential role of leptin in diverse skin diseases.

\begin{tabular}{|c|c|c|c|}
\hline Skin diseases & Serum level of leptin & Potential effect & References \\
\hline Psoriasis & Elevated & 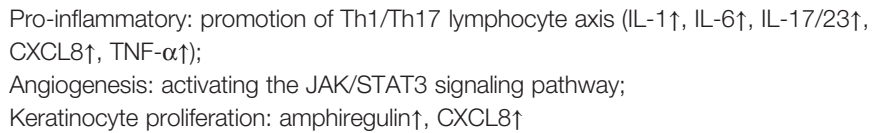 & $(55-69)$ \\
\hline Systemic sclerosis & $\begin{array}{l}\text { Not significantly changed/ } \\
\text { Elevated }\end{array}$ & Anti-fibrotic: inhibiting the JAK/STAT3 signaling pathway & $\begin{array}{l}(71-73) \\
(74-75)\end{array}$ \\
\hline Systemic lupus erythematosus & Elevated & $\begin{array}{l}\text { Pro-inflammatory: promotion of the Th1/Th17 lymphocyte axis, inhibition of Treg } \\
\text { lymphocytes; } \\
\text { Aging of mesenchymal stem cells (via the NAP-2 and PI3K-Akt pathway) }\end{array}$ & $(79-86)$ \\
\hline $\begin{array}{l}\text { Melanoma and non-pigment } \\
\text { tumors }\end{array}$ & Elevated & 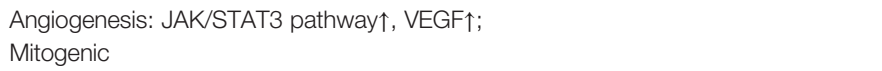 & $(90-94)$ \\
\hline Skin tags & Elevated & Promotion of keratinocyte and fibroblast growth & $(95,96)$ \\
\hline Hidradenitis suppurativa & Elevated & Pro-inflammatory: promotion of Th1 lymphocytes & $(97,98)$ \\
\hline
\end{tabular}




\section{AUTHOR CONTRIBUTIONS}

XS and DC contributed to the study design. XS wrote the manuscript. All authors contributed to the article and approved the submitted version.

\section{REFERENCES}

1. Su X, Luo M, Tang X, Luo Y, Zheng X, Peng D, et al. Goals of non-high density lipoprotein cholesterol need to be adjusted in Chinese acute coronary syndrome patients: Findings from the CCC-ACS project. Clin Chim Acta (2019) 496:48-54. doi: 10.1016/j.cca.2019.06.022

2. Su X, Kong Y, Peng D. Evidence for changing lipid management strategy to focus on non-high density lipoprotein cholesterol. Lipids Health Dis (2019) 18:134. doi: 10.1186/s12944-019-1080-x

3. Su X, Peng D. New insight into sortilin in controlling lipid metabolism and the risk of atherogenesis. Biol Rev Camb Philos Soc (2019) 95:232-243. doi: 10.1111/brv.12561

4. Su X, Peng D. The exchangeable apolipoproteins in lipid metabolism and obesity. Clin Chim Acta (2020) 503:128-35. doi: 10.1016/j.cca.2020.01.015

5. Munzberg H, Morrison CD. Structure, production and signaling of leptin. Metabolism (2015) 64:13-23. doi: 10.1016/j.metabol.2014.09.010

6. Poeggeler B, Schulz C, Pappolla MA, Bodo E, Tiede S, Lehnert H, et al. Leptin and the skin: a new frontier. Exp Dermatol (2010) 19:12-8. doi: 10.1111/ j.1600-0625.2009.00930.x

7. Denver RJ, Bonett RM, Boorse GC. Evolution of leptin structure and function. Neuroendocrinology (2011) 94:21-38. doi: 10.1159/000328435

8. Seremet S, Gurel MS. Miscellaneous skin disease and the metabolic syndrome. Clin Dermatol (2018) 36:94-100. doi: 10.1016/j.clindermatol.2017.09.016

9. Unlu B, Tursen U. Autoimmune skin diseases and the metabolic syndrome. Clin Dermatol (2018) 36:67-71. doi: 10.1016/j.clindermatol.2017.09.012

10. Saltiel AR, Olefsky JM. Inflammatory mechanisms linking obesity and metabolic disease. J Clin Invest (2017) 127:1-4. doi: 10.1172/JCI92035

11. Makki K, Froguel P, Wolowczuk I. Adipose tissue in obesity-related inflammation and insulin resistance: cells, cytokines, and chemokines. ISRN Inflammation (2013) 2013:139239. doi: 10.1155/2013/139239

12. Tobin AM, Ahern T, Rogers S, Collins P, O'Shea D, Kirby B. The dermatological consequences of obesity. Int J Dermatol (2013) 52:927-32. doi: $10.1111 /$ j.1365-4632.2012.05624.x

13. Kuroda M, Sakaue H. Adipocyte Death and Chronic Inflammation in Obesity. J Med Invest (2017) 64:193-6. doi: 10.2152/jmi.64.193

14. Hirt PA, Castillo DE, Yosipovitch G, Keri JE. Skin changes in the obese patient. J Am Acad Dermatol (2019) 81:1037-57. doi: 10.1016/ j.jaad.2018.12.070

15. Velazquez-Bautista M, Lopez-Sandoval JJ, Gonzalez-Hita M, Vazquez-Valls E, Cabrera-Valencia IZ, Torres-Mendoza BM. Association of metabolic syndrome with low birth weight, intake of high-calorie diets and acanthosis nigricans in children and adolescents with overweight and obesity. Endocrinol Diabetes Nutr (2017) 64:11-7. doi: 10.1016/j.endien.2016.09.002

16. Kong Y, Zhang S, Wu R, Su X, Peng D, Zhao M, et al. New insights into different adipokines in linking the pathophysiology of obesity and psoriasis. Lipids Health Dis (2019) 18:171. doi: 10.1186/s12944-019-1115-3

17. Mahieu MA, Ahn GE, Chmiel JS, Dunlop DD, Helenowski IB, Semanik P, et al. Serum adipokine levels and associations with patient-reported fatigue in systemic lupus erythematosus. Rheumatol Int (2018) 38:1053-61. doi: 10.1007/s00296-017-3924-x

18. Van Doorn C, Macht VA, Grillo CA, Reagan LP. Leptin resistance and hippocampal behavioral deficits. Physiol Behav (2017) 176:207-13. doi: 10.1016/j.physbeh.2017.03.002

19. Crujeiras AB, Carreira MC, Cabia B, Andrade S, Amil M, Casanueva FF. Leptin resistance in obesity: An epigenetic landscape. Life Sci (2015) 140:5763. doi: 10.1016/j.lfs.2015.05.003

20. Bates SH, Stearns WH, Dundon TA, Schubert M, Tso AW, Wang Y, et al. STAT3 signalling is required for leptin regulation of energy balance but not reproduction. Nature (2003) 421:856-9. doi: 10.1038/nature01388

\section{FUNDING}

This work was supported by grants from the National Key Research and Development Program of China (no. 2016YFC1301202).

21. Jequier E. Leptin signaling, adiposity, and energy balance. Ann N Y Acad Sci (2002) 967:379-88. doi: 10.1111/j.1749-6632.2002.tb04293.x

22. Michalska-Jakubus M, Sawicka K, Potembska E, Kowal M, Krasowska D. Clinical associations of serum leptin and leptin/adiponectin ratio in systemic sclerosis. Postepy Dermatol Alergol (2019) 36:325-38. doi: 10.5114/ ada.2018.75809

23. Liu J, Yang X, Yu S, Zheng R. The Leptin Signaling. Adv Exp Med Biol (2018) 1090:123-44. doi: 10.1007/978-981-13-1286-1_7

24. Wada N, Hirako S, Takenoya F, Kageyama H, Okabe M, Shioda S. Leptin and its receptors. J Chem Neuroanat (2014) 61-62:191-9. doi: 10.1016/ j.jchemneu.2014.09.002

25. Schaab M, Kratzsch J. The soluble leptin receptor. Best Pract Res Clin Endocrinol Metab (2015) 29:661-70. doi: 10.1016/j.beem.2015.08.002

26. Wauman J, Zabeau L, Tavernier J. The Leptin Receptor Complex: Heavier Than Expected? Front Endocrinol (Lausanne) (2017) 8:30. doi: 10.3389/ fendo.2017.00030

27. Zhang Y, Chua S Jr. Leptin Function and Regulation. Compr Physiol (2017) 8:351-69. doi: 10.1002/cphy.c160041

28. Kwon O, Kim KW, Kim MS. Leptin signalling pathways in hypothalamic neurons. Cell Mol Life Sci (2016) 73:1457-77. doi: 10.1007/s00018-016-2133-1

29. Yadav A, Kataria MA, Saini V, Yadav A. Role of leptin and adiponectin in insulin resistance. Clin Chim Acta (2013) 417:80-4. doi: 10.1016/ j.cca.2012.12.007

30. Paz-Filho G, Mastronardi CA, Licinio J. Leptin treatment: facts and expectations. Metabolism (2015) 64:146-56. doi: 10.1016/ j.metabol.2014.07.014

31. Gulcelik NE, Halil M, Ariogul S, Usman A. Adipocytokines and aging: adiponectin and leptin. Minerva Endocrinol (2013) 38:203-10.

32. La Cava A. Leptin in inflammation and autoimmunity. Cytokine (2017) 98:51-8. doi: 10.1016/j.cyto.2016.10.011

33. Stofkova A. Leptin and adiponectin: from energy and metabolic dysbalance to inflammation and autoimmunity. Endocr Regul (2009) 43:157-68.

34. Abella V, Scotece M, Conde J, Pino J, Gonzalez-Gay MA, Gomez-Reino JJ, et al. Leptin in the interplay of inflammation, metabolism and immune system disorders. Nat Rev Rheumatol (2017) 13:100-9. doi: 10.1038/ nrrheum.2016.209

35. Lee M, Lee E, Jin SH, Ahn S, Kim SO, Kim J, et al. Leptin regulates the proinflammatory response in human epidermal keratinocytes. Arch Dermatol Res (2018) 310:351-62. doi: 10.1007/s00403-018-1821-0

36. Murad A, Nath AK, Cha ST, Demir E, Flores-Riveros J, Sierra-Honigmann MR. Leptin is an autocrine/paracrine regulator of wound healing. FASEB J (2003) 17:1895-7. doi: 10.1096/fj.03-0068fje

37. Glasow A, Kiess W, Anderegg U, Berthold A, Bottner A, Kratzsch J. Expression of leptin $(\mathrm{Ob})$ and leptin receptor $(\mathrm{Ob}-\mathrm{R})$ in human fibroblasts: regulation of leptin secretion by insulin. J Clin Endocrinol Metab (2001) 86:4472-9. doi: 10.1210/jcem.86.9.7792

38. Tadokoro S, Ide S, Tokuyama R, Umeki H, Tatehara S, Kataoka S, et al. Leptin promotes wound healing in the skin. PloS One (2015) 10:e0121242. doi: 10.1371/journal.pone.0121242

39. Kanda N, Watanabe S. Leptin enhances human beta-defensin-2 production in human keratinocytes. Endocrinology (2008) 149:5189-98. doi: 10.1210/ en.2008-0343

40. Frank S, Stallmeyer B, Kampfer H, Kolb N, Pfeilschifter J. Leptin enhances wound re-epithelialization and constitutes a direct function of leptin in skin repair. J Clin Invest (2000) 106:501-9. doi: 10.1172/JCI9148

41. Tong KM, Shieh DC, Chen CP, Tzeng CY, Wang SP, Huang KC, et al. Leptin induces IL-8 expression via leptin receptor, IRS-1, PI3K, Akt cascade and promotion of NF-kappaB/p300 binding in human synovial fibroblasts. Cell Signal (2008) 20:1478-88. doi: 10.1016/j.cellsig.2008.04.003 
42. Kim YJ, Kim JS, Seo YR, Park JH, Choi MS, Sung MK. Carnosic acid suppresses colon tumor formation in association with antiadipogenic activity. Mol Nutr Food Res (2014) 58:2274-85. doi: 10.1002/mnfr.201400293

43. Sumikawa Y, Inui S, Nakajima T, Itami S. Hair cycle control by leptin as a new anagen inducer. Exp Dermatol (2014) 23:27-32. doi: 10.1111/exd.12286

44. Won CH, Yoo HG, Kwon OS, Sung MY, Kang YJ, Chung JH, et al. Hair growth promoting effects of adipose tissue-derived stem cells. J Dermatol Sci (2010) 57:134-7. doi: 10.1016/j.jdermsci.2009.10.013

45. Watabe R, Yamaguchi T, Kabashima-Kubo R, Yoshioka M, Nishio D, Nakamura M. Leptin controls hair follicle cycling. Exp Dermatol (2014) 23:228-9. doi: 10.1111/exd.12335

46. Lebwohl MG, Bachelez H, Barker J, Girolomoni G, Kavanaugh A, Langley RG, et al. Patient perspectives in the management of psoriasis: results from the population-based Multinational Assessment of Psoriasis and Psoriatic Arthritis Survey. J Am Acad Dermatol (2014) 70:871-81 e1-30. doi: 10.1016/j.jaad.2013.12.018

47. Parisi R, Symmons DP, Griffiths CE, Ashcroft DM, Identification PManagement of, and t. Associated ComorbidiTy project, Global epidemiology of psoriasis: a systematic review of incidence and prevalence. J Invest Dermatol (2013) 133:377-85. doi: 10.1038/jid.2012.339

48. Russell TJ, Schultes LM, Kuban DJ. Histocompatibility (HL-A) antigens associated with psoriasis. N Engl J Med (1972) 287:738-40. doi: 10.1056/ NEJM197210122871503

49. Barrea L, Nappi F, Di Somma C, Savanelli MC, Falco A, Balato A, et al. Environmental Risk Factors in Psoriasis: The Point of View of the Nutritionist. Int J Environ Res Public Health (2016) 13:743-49. doi: 10.3390/ijerph13070743

50. Naldi L, Chatenoud L, Linder D, Belloni Fortina A, Peserico A, Virgili AR, et al. Cigarette smoking, body mass index, and stressful life events as risk factors for psoriasis: results from an Italian case-control study. $J$ Invest Dermatol (2005) 125:61-7. doi: 10.1111/j.0022-202X.2005.23681.x

51. Zeng J, Luo S, Huang Y, Lu Q. Critical role of environmental factors in the pathogenesis of psoriasis. J Dermatol (2017) 44:863-72. doi: 10.1111/13468138.13806

52. Owen CM, Chalmers RJ, O'Sullivan T, Griffiths CE. A systematic review of antistreptococcal interventions for guttate and chronic plaque psoriasis. $\mathrm{Br} \mathrm{J}$ Dermatol (2001) 145:886-90. doi: 10.1046/j.1365-2133.2001.04504.x

53. Rachakonda TD, Dhillon JS, Florek AG, Armstrong AW. Effect of tonsillectomy on psoriasis: a systematic review. J Am Acad Dermatol (2015) 72:261-75. doi: 10.1016/j.jaad.2014.10.013

54. Coimbra S, Oliveira H, Reis F, Belo L, Rocha S, Quintanilha A, et al. Circulating adipokine levels in Portuguese patients with psoriasis vulgaris according to body mass index, severity and therapy. J Eur Acad Dermatol Venereol (2010) 24:1386-94. doi: 10.1111/j.1468-3083.2010.03647.x

55. Kyriakou A, Patsatsi A, Sotiriadis D, Goulis DG. Serum Leptin, Resistin, and Adiponectin Concentrations in Psoriasis: A Meta-Analysis of Observational Studies. Dermatology (2017) 233:378-89. doi: 10.1159/000481882

56. Myers MG Jr., Leibel RL, Seeley RJ, Schwartz MW. Obesity and leptin resistance: distinguishing cause from effect. Trends Endocrinol Metab (2010) 21:643-51. doi: 10.1016/j.tem.2010.08.002

57. Mitsuyama S, Abe F, Kimura M, Yoshida M, Higuchi T. Association between leptin gene expression in subcutaneous adipose tissue and circulating leptin levels in obese patients with psoriasis. Arch Dermatol Res (2015) 307:539-44. doi: 10.1007/s00403-015-1581-z

58. Campanati A, Ganzetti G, Giuliodori K, Marra M, Bonfigli A, Testa R, et al. Serum levels of adipocytokines in psoriasis patients receiving tumor necrosis factor-alpha inhibitors: results of a retrospective analysis. Int J Dermatol (2015) 54:839-45. doi: 10.1111/ijd.12706

59. Cerman AA, Bozkurt S, Sav A, Tulunay A, Elbasi MO, Ergun T. Serum leptin levels, skin leptin and leptin receptor expression in psoriasis. Br J Dermatol (2008) 159:820-6. doi: 10.1111/j.1365-2133.2008.08742.x

60. Stjernholm T, Ommen P, Langkilde A, Johansen C, Iversen L, Rosada C, et al. Leptin deficiency in mice counteracts imiquimod (IMQ)-induced psoriasislike skin inflammation while leptin stimulation induces inflammation in human keratinocytes. Exp Dermatol (2017) 26:338-45. doi: 10.1111/exd.13149

61. Shimoura N, Nagai H, Fujiwara S, Jimbo H, Nishigori C. Exacerbation and Prolongation of Psoriasiform Inflammation in Diabetic Obese Mice: A Synergistic Role of CXCL5 and Endoplasmic Reticulum Stress. J Invest Dermatol (2018) 138:854-63. doi: 10.1016/j.jid.2017.10.023
62. Johnston A, Arnadottir S, Gudjonsson JE, Aphale A, Sigmarsdottir AA, Gunnarsson SI, et al. Obesity in psoriasis: leptin and resistin as mediators of cutaneous inflammation. Br J Dermatol (2008) 159:342-50. doi: 10.1111/ j.1365-2133.2008.08655.x

63. Fritz Y, Klenotic PA, Swindell WR, Yin ZQ, Groft SG, Zhang L, et al. Induction of Alternative Proinflammatory Cytokines Accounts for Sustained Psoriasiform Skin Inflammation in IL-17C+IL-6KO Mice. J Invest Dermatol (2017) 137:696-705. doi: 10.1016/j.jid.2016.10.021

64. Calautti E, Avalle L, Poli V. Psoriasis: A STAT3-Centric View. Int J Mol Sci (2018) 19:171-78. doi: 10.3390/ijms19010171

65. Markaki A, Gkouskou K, Stylianou K, Dermitzaki E, Perakis K, Margioris A, et al. Relationship between adiposity, adipokines, inflammatory markers and lipid profile in hemodialysis patients. Eur Rev Med Pharmacol Sci (2014) 18:1496-8.

66. Mauro CR, Nguyen BT, Yu P, Tao M, Gao I, Seidman MA, et al. Inflammatory "adiposopathy" in major amputation patients. Ann Vasc Surg (2013) 27:34652. doi: 10.1016/j.avsg.2012.07.017

67. Watanabe K, Suzukawa M, Arakawa S, Kobayashi K, Igarashi S, Tashimo H, et al. Leptin enhances cytokine/chemokine production by normal lung fibroblasts by binding to leptin receptor. Allergol Int (2019) 68S:S3-8. doi: 10.1016/j.alit.2019.04.002

68. Torres T, Bettencourt N, Ferreira J, Carvalho C, Mendonca D, Vasconcelos C, et al. Lack of association between leptin, leptin receptor, adiponectin gene polymorphisms and epicardial adipose tissue, abdominal visceral fat volume and atherosclerotic burden in psoriasis patients. Arch Physiol Biochem (2015) 121:103-8. doi: 10.3109/13813455.2015.1024136

69. Karpouzis A, Tripsianis G, Gatzidou E, Veletza S. Assessment of Leptin Gene Polymorphism rs2060713 in Psoriasis Vulgaris. ISRN Dermatol (2014) 2014:845272. doi: 10.1155/2014/845272

70. Abdel Hay RM, Rashed LA. Association between the leptin gene 2548G/A polymorphism, the plasma leptin and the metabolic syndrome with psoriasis. Exp Dermatol (2011) 20:715-9. doi: 10.1111/j.1600-0625.2011.01299.x

71. Harada K, Maeda T, Matsubayashi J, Uchiyama M, Irisawa R, Go K, et al. Centrifugal lipodystrophy of the scalp manifesting as centrifugal lipodystrophic alopecia. Clin Exp Dermatol (2018) 43:286-90. doi: 10.1111/ ced. 13328

72. Budulgan M, Dilek B, Dag SB, Batmaz I, Yildiz I, Sariyildiz MA, et al. Relationship between serum leptin level and disease activity in patients with systemic sclerosis. Clin Rheumatol (2014) 33:335-9. doi: 10.1007/s10067-0132459-0

73. Olewicz-Gawlik A, Danczak-Pazdrowska A, Kuznar-Kaminska B, BaturaGabryel H, Katulska K, Wojciech S, et al. Circulating adipokines and organ involvement in patients with systemic sclerosis. Acta Reumatol Port (2015) 40:156-62.

74. Zhao JH, Huang XL, Duan Y, Wang YJ, Chen SY, Wang J. Serum adipokines levels in patients with systemic sclerosis: A meta-analysis. Mod Rheumatol (2017) 27:298-305. doi: 10.1080/14397595.2016.1193106

75. Pehlivan Y, Onat AM, Ceylan N, Turkbeyler IH, Buyukhatipoglu H, Comez G, et al. Serum leptin, resistin and TNF-alpha levels in patients with systemic sclerosis: the role of adipokines in scleroderma. Int J Rheumatol Dis (2012) 15:374-9. doi: 10.1111/j.1756-185X.2012.01755.x

76. Stochmal A, Czuwara J, Zaremba M, Rudnicka L. Altered serum level of metabolic and endothelial factors in patients with systemic sclerosis. Arch Dermatol Res (2020) 312:453-8. doi: 10.1007/s00403-019-01993-y

77. De Rosa V, Procaccini C, Cali G, Pirozzi G, Fontana S, Zappacosta S, et al. A key role of leptin in the control of regulatory $\mathrm{T}$ cell proliferation. Immunity (2007) 26:241-55. doi: 10.1016/j.immuni.2007.01.011

78. Gonzalez M, Lind L, Soderberg S. Leptin and endothelial function in the elderly: the Prospective Investigation of the Vasculature in Uppsala Seniors (PIVUS) study. Atherosclerosis (2013) 228:485-90. doi: 10.1016/ j.atherosclerosis.2013.03.018

79. Li Q, Wu H, Liao W, Zhao M, Chan V, Li L, et al. A comprehensive review of immune-mediated dermatopathology in systemic lupus erythematosus. $J$ Autoimmun (2018) 93:1-15. doi: 10.1016/j.jaut.2018.07.007

80. Barbosa Vde S, Francescantonio PL, Silva NA. Leptin and adiponectin in patients with systemic lupus erythematosus: clinical and laboratory correlations. Rev Bras Reumatol (2015) 55:140-5. doi: 10.1016/ j.rbre.2014.08.013 
81. Demir S, Erten G, Artim-Esen B, Sahinkaya Y, Pehlivan O, Alpay-Kanitez N, et al. Increased serum leptin levels are associated with metabolic syndrome and carotid intima media thickness in premenopausal systemic lupus erythematosus patients without clinical atherosclerotic vascular events. Lupus (2018) 27:1509-16. doi: 10.1177/0961203318782424

82. Lee YH, Song GG. Association between circulating leptin levels and systemic lupus erythematosus: an updated meta-analysis. Lupus (2018) 27:428-35. doi: $10.1177 / 0961203317725587$

83. Xu WD, Zhang M, Zhang YJ, Liu SS, Pan HF, Ye DQ. Association between leptin and systemic lupus erythematosus. Rheumatol Int (2014) 34:559-63. doi: 10.1007/s00296-013-2774-4

84. Yu Y, Liu Y, Shi FD, Zou H, Matarese G, La Cava A. Cutting edge: Leptininduced RORgammat expression in CD4+ T cells promotes Th17 responses in systemic lupus erythematosus. J Immunol (2013) 190:3054-8. doi: 10.4049/ jimmunol.1203275

85. Chen H, Shi B, Feng X, Kong W, Chen W, Geng L, et al. Leptin and Neutrophil-Activating Peptide 2 Promote Mesenchymal Stem Cell Senescence Through Activation of the Phosphatidylinositol 3-Kinase/Akt Pathway in Patients With Systemic Lupus Erythematosus. Arthritis Rheumatol (2015) 67:2383-93. doi: 10.1002/art.39196

86. Amarilyo G, Iikuni N, Shi FD, Liu A, Matarese G, La Cava A. Leptin promotes lupus T-cell autoimmunity. Clin Immunol (2013) 149:530-3. doi: 10.1016/ j.clim.2013.09.002

87. Yuan Q, Chen H, Li X, Wei J. Leptin: an unappreciated key player in SLE. Clin Rheumatol (2020) 39:305-17. doi: 10.1007/s10067-019-04831-8

88. Al M, Ng L, Tyrrell P, Bargman J, Bradley T, Silverman E. Adipokines as novel biomarkers in paediatric systemic lupus erythematosus. Rheumatol (Oxford) (2009) 48:497-501. doi: 10.1093/rheumatology/kep030

89. Li HM, Zhang TP, Leng RX, Li XP, Li XM, Pan HF. Plasma/Serum Leptin Levels in Patients with Systemic Lupus Erythematosus: A Meta-analysis. Arch Med Res (2015) 46:551-6. doi: 10.1016/j.arcmed.2015.09.005

90. Vadacca M, Zardi EM, Margiotta D, Rigon A, Cacciapaglia F, Arcarese L, et al. Leptin, adiponectin and vascular stiffness parameters in women with systemic lupus erythematosus. Intern Emerg Med (2013) 8:705-12. doi: 10.1007/ s11739-011-0726-0

91. Amjadi F, Mehdipoor R, Zarkesh-Esfahani H, Javanmard SH. Leptin serves as angiogenic/mitogenic factor in melanoma tumor growth. Adv BioMed Res (2016) 5:127. doi: 10.4103/2277-9175.187005
92. El Safoury OS, Abdel Hay RM, Fawzy MM, Kadry D, Amin IM, Abu Zeid OM, et al. Skin tags, leptin, metabolic syndrome and change of the life style. Indian J Dermatol Venereol Leprol (2011) 77:577-80. doi: 10.4103/0378-6323.84061

93. Praestegaard C, Kjaer SK, Christensen J, Tjonneland A, Halkjaer J, Jensen A. Obesity and risks for malignant melanoma and non-melanoma skin cancer: results from a large Danish prospective cohort study. J Invest Dermatol (2015) 135:901-4. doi: 10.1038/jid.2014.438

94. Rivadeneira DB, DePeaux K, Wang Y, Kulkarni A, Tabib T, Menk AV, et al. Oncolytic Viruses Engineered to Enforce Leptin Expression Reprogram Tumor-Infiltrating T Cell Metabolism and Promote Tumor Clearance. Immunity (2019) 51:548-560 e4. doi: 10.1016/j.immuni.2019.07.003

95. Zhou B, Wu D, Liu H, Du LT, Wang YS, Xu JW, et al. Obesity and pancreatic cancer: An update of epidemiological evidence and molecular mechanisms. Pancreatology (2019) 19:941-50. doi: 10.1016/j.pan.2019.08.008

96. Putra IB, Siregar R, Jusuf NK, Ginting O, Nurhayati R. Correlation between Serum Leptin Level with Type and Number of Lesion Skin Tag. Open Access Maced J Med Sci (2019) 7:53-5. doi: 10.3889/oamjms.2019.020

97. Shaheen MA, Abdel Fattah NS, Sayed YA, Saad AA. Assessment of serum leptin, insulin resistance and metabolic syndrome in patients with skin tags. $J$ Eur Acad Dermatol Venereol (2012) 26:1552-7. doi: 10.1111/j.14683083.2011.04401.x

98. Malara A, Hughes R, Jennings L, Sweeney CM, Lynch M, Awdeh F, et al. Adipokines are dysregulated in patients with hidradenitis suppurativa. $\mathrm{Br} J$ Dermatol (2018) 178:792-3. doi: 10.1111/bjd.15904

99. Shalom G, Freud T, Harman-Boehm I, Polishchuk I, Cohen AD. Hidradenitis suppurativa and metabolic syndrome: a comparative cross-sectional study of 3207 patients. Br J Dermatol (2015) 173:464-70. doi: 10.1111/bjd.13777

Conflict of Interest: The authors declare that the research was conducted in the absence of any commercial or financial relationships that could be construed as a potential conflict of interest.

Copyright (C) 2021 Su, Cheng and Chang. This is an open-access article distributed under the terms of the Creative Commons Attribution License (CC BY). The use, distribution or reproduction in other forums is permitted, provided the original author(s) and the copyright owner(s) are credited and that the original publication in this journal is cited, in accordance with accepted academic practice. No use, distribution or reproduction is permitted which does not comply with these terms. 